\title{
INFECÇÃO EM IDOSOS INTERNADOS EM INSTITUIÇÃO DE LONGA PERMANÊNCIA
}

Paulo José Fortes Villas Bôas*, Ana lúcia dos Anjos Ferreira

Trabalho realizado no Departamento de Clínica Médica da Faculdade de Medicina de Botucatu - Unesp, SP

* Correspondência:

Rua Cardoso de Almeida,

1807

Botucatu/SP

Cep: 18600-005

\section{RESUMO}

OBJETIVO. Avaliar ocorrência de infecção em idosos de instituiç̧ão de longa permanência (ILP) durante I3 meses consecutivos. Métodos. Cinqüenta e cinco idosos foram avaliados seģundo critérios de infecção e de comunicação padronizada. Resultados. A idade mediana foi de 75 (66-8I) anos. O conjunto de dados constituiu-se de 20.896 pacientes-dia (PD) durante o período, com um total de 76 infecçōes para uma taxa média de infecção de 3,2 por 1000 PD. A taxa de pacientes com infecção foi de $63,6 \%$. As topografias prevalentes foram respiratória (50\%), trato urinário (32\%), pele e partes moles (12\%) e gastrintestinal (7\%), com taxa de infecção/l000 PD de I,60; I,01; 0,38; e 0,2, respectivamente. A taxa de mortalidade por infecção foi de 5\%. Agentes microbilóogicos foram isolados em 30,5\% dos episódios infecciosos e Escherichia coli e Staphylococcus aureus foram os mais freqüentes.

Conclusão. Infecção apresentou elevada incidência nos idosos institucionalizados, porém nenhum evento extraordinário foi observado.

Unitermos: Idoso. Infecção. Epidemiologia. Instituiç̧ão de longa permanência.

\section{INTRODUÇÃO}

O envelhecimento populacional constitui a mais importante mudança demográfica tanto em países desenvolvidos quanto em países em desenvolvimento, como o Brasil. A Organização Mundial da Saúde propõe 60 anos ou mais como idade em estudos da população idosa'.

Uma importante conseqüência do envelhecimento populacional é o aumento de idosos com dependência e, conseqüentemente, com necessidade de institucionalização. Estima-se que $40 \%$ das pessoas com 65 anos ou mais necessitarão de cuidados em uma instituição de longa permanência (ILP) durante a sua vida. Projeções na população americana mostram que o número de pessoas com necessidade dessa assistência aumentará de 1,5 para 5 milhões no ano de 2030². Estimou-se que 0,6\% a 1,3\% da população brasileira idosa encontravam-se em ILP3.

Em comparação a indivíduos jovens, os idosos apresentam diversos eventos que os predispõem à infecção, condição que aumenta a morbidade e mortalidade ${ }^{4-6}$. Os residentes das ILP, por apresentarem maior grau de dependência e de doenças clínicas (em média três diagnósticos de doenças crônicas) que os idosos que vivem na comunidade, apresentam maior risco de adquirir doença infecciosa ${ }^{4}$. Esses processos são importantes causas de mortalidade, declínio cognitivo e incapacidade que acometem essa faixa etária.

O processo infeccioso é uma das mais freqüentes causas de hospitalização e de morte em pacientes em ILP7. Levantamentos de prevalência de infecções em unidades geriátricas mostram que $5 \%$ a 10\% dos pacientes desenvolvem algum quadro no decorrer de um mês. O Centro de Controle de Doenças (CDC-EUA) calcula que ocorram por ano 1,5 milhão de infecções nos institucionalizados, que corresponde a uma infecção por residente ao $a n o^{5,8}$, em média.

O presente estudo teve por finalidade conhecer a ocorrência das infecções em uma instituição de longa permanência, pois poucos trabalhos avaliam tal situação nessa população específica.

\section{Métodos}

Após aprovação pelo Comitê de Ética em Pesquisa da Faculdade de Medicina de Botucatu / Unesp (Ofício no 272/2003), os indivíduos institucionalizados no Asilo Padre Euclides de Botucatu foram avaliados no período de agosto de 2003 a agosto de 2004. O paciente concordou em participar do estudo por meio de leitura e assinatura do termo de consentimento informado e esclarecido que the foi fornecido, ou ao responsável pela instituição, no momento da avaliação inicial.

As infecções foram classificadas entre as diversas topografias utilizando as definições padronizadas pela Society for Healthcare Epidemiology of America (SHEA) e American Geriatrics Society, que incluem critérios clínicos e laboratoriais, 6.9 .

Os pacientes foram avaliados com visitas à ILP por equipe de enfermagem devidamente treinada para avaliar o agravo à saúde dessa população. $\mathrm{Na}$ evidência de episódio sugestivo de infecção, a equipe médica foi acionada para avaliações clínica, laboratorial e radiológica.

Os exames laboratoriais e radiológicos, quando necessários, foram realizados no Hospital das Clínicas da Faculdade de Medicina de Botucatu / Unesp.

Os dados obtidos das avaliações foram inicialmente descritos 
INFECÇÃO EM IDOSOS INTERNADOS EM INSTITUIÇÃO DE LONGA PERMANÊNCIA

em termos de variáveis quantitativas discretas ou contínuas e transcritos em instrumento desenvolvido para essa finalidade.

O programa estatístico utilizado para o armazenamento e análise dos dados foi o SPSS para Windows Versão 12.0.

Foram calculados ocorrência mensal de infecção; densidade de incidência de infecção (episódio novo de infecção x 1000 / número de pacientes-dia no período); taxa de infecção (número de pacientes que cursaram com infecção / número total de institucionalizados); distribuição percentual das topografias de infecção; taxa de mortalidade; taxa de letalidade por infecção; e agentes etiológicos das infecções.

\section{Resultados}

Foram acompanhados 55 idosos da ILP, sendo 20 homens e 35 mulheres. A população estudada apresentava idade mediana de 75 anos (percentil 25 (p25) de 66 e percentil 75 (p75) de 8I). A idade mediana do grupo feminino foi de 75 e a do masculino de 74,5 anos $(p=0,87)$. No início do estudo, a mediana do tempo de institucionalização era de 6 (p25 de 1,2 e p75 de 14) anos, sendo de $4 \pm 4,5$ anos e $7 \pm 3,2$ para o grupo masculino e feminino, respectivamente $(p=0,29)$.

Em média, cada idoso possuía 3,4 ( $\pm 2,3)$ diagnósticos clínicos, sendo os mais freqüentes: história prévia ou atual de tabagismo (45\%), hipertensão arterial (45\%), dependência para as atividades instrumentais de vida diária (AIVD) (45\%), baixo peso, considerado com índice de massa corporal $<22 \mathrm{~kg} / \mathrm{m}^{2}$ (40\%), sobrepeso (IMC>27) (30\%) e dependência para atividade básica de vida diária (25,4\%).

Foram coletados dados de 20.896 pacientes-dia. Ocorreram 72 episódios de infecção em 35 institucionalizados, com a distribuição mensal apresentada no Gráfico I, sendo que os episódios ocorreram durante todo o período do estudo. As maiores prevalências foram observadas nos períodos de agosto a outubro de 2003 (19 episódios) e abril a junho de 2004 (32 episódios). A taxa de pacientes com infecção foi de 63,6\%. A densidade de infecção foi de 3,4 infecções por 1000 pacientes-dia (72 episódios de infecção x 1000 / 20.896 pacientes-dia). Internação hospitalar foi necessária em oito (1 1\%) episódios de infecção. Ocorreram cinco óbitos por infecção, relacionada ao quadro respiratório, que determinou uma taxa de mortalidade dos institucionalizados por infecção de $9 \%$ (cinco óbitos em 55 pacientes) e de letalidade por infecção de 6,9\% (cinco óbitos em 72 episódios).

A Tabela I apresenta a distribuição percentual das topografias das infecções, sendo as mais prevalentes: respiratória (50\%), trato urinário (32\%), pele e partes moles (11\%). Das infecções respiratórias, destaca-se que 21 (28,9\%) originaram-se de vias aéreas superiores e $15(21,1 \%)$ das inferiores (pneumonia).

Agentes etiológicos foram isolados em 23 episódios de infecção (31,9\%), sendo os mais freqüentes: Escherichia coli, Staphylococcus coagulase negativa e Pseudomonas aeruginosa. A Tabela 2 mostra a distribuição dos agentes etiológicos por topografia de infecção. Por dificuldades técnicas não foram coletadas amostras de secreção do trato respiratório.

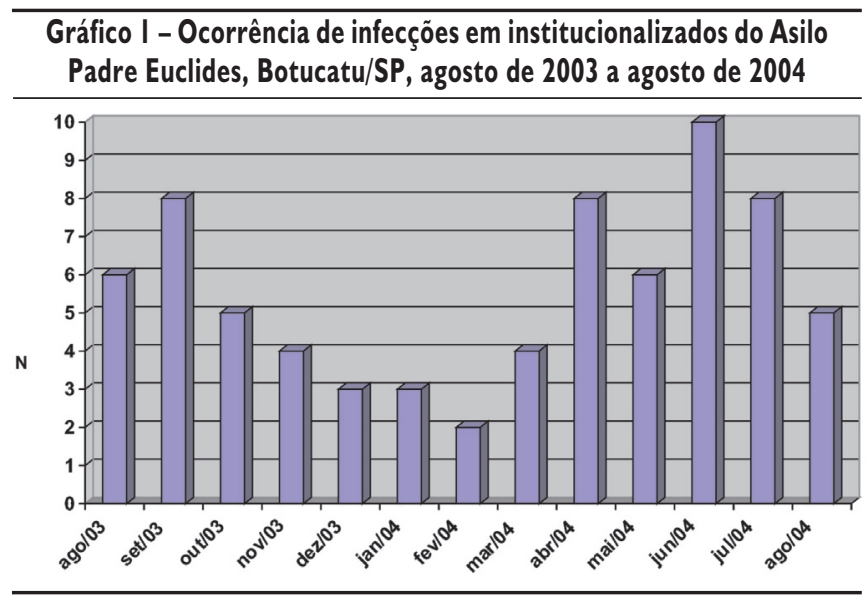

\begin{tabular}{|c|c|c|}
\hline Topografia & Episódio (n) & $\%$ \\
\hline Respiratória & 36 & 50 \\
\hline Urinária & 23 & 3 \\
\hline Pele e partes moles & 8 & II \\
\hline Gastrintestinal & 5 & 7 \\
\hline Total & 72 & 100 \\
\hline
\end{tabular}

\begin{tabular}{|c|c|c|}
\hline \multicolumn{3}{|c|}{$\begin{array}{l}\text { Tabela 2- Distribuição dos agentes etiológicos por topografia de } \\
\text { infecção em institucionalizados do Asilo Padre Euclides, } \\
\text { Botucatu/SP, agosto de } 2003 \text { a agosto de } 2004\end{array}$} \\
\hline Topografia & $\mathbf{N}$ & Agente \\
\hline Urinária & 18 & $\begin{array}{c}\text { Escherichia coli ( I I) } \\
\text { Staphylococcus coagulase negativa (4) } \\
\text { Pseudomonas aeruginosa (3) }\end{array}$ \\
\hline Pele e partes moles & 4 & $\begin{array}{c}\text { Staphylococcus coagulase negativa (2) } \\
\text { Pseudomonas aeruginosa (2) }\end{array}$ \\
\hline Gastrintestinal & | & Escherichia coli (I) \\
\hline
\end{tabular}

\section{Discussão}

Quando comparado com o jovem e o idoso, em particular, o institucionalizado está mais suscetível à infecção em razão de alterações fisiológicas do envelhecimento, declínio da resposta imunológica e a presença de doenças concomitantes ${ }^{5,6}$, com conseqüente aumento de morbidade e mortalidade ${ }^{5}$. São várias as alterações anatômicas e funcionais que colaboram para o entendimento dos mecanismos que predispõem essa população à infecção $0^{5}$. $O$ declínio na função do linfócito $T$ e na imunidade celular e a diminuição da produção de anticorpos na imunização extrínseca são alterações imunológicas que podem aumentar a vulnerabilidade à infecçã̃o. $O$ adelgaçamento da pele e o menor reflexo da tosse são 
BÔAS PJFV ET AL.

alterações fisiológicas do envelhecimento que facilitam a infecção de pele e partes moles e à aspiração e pneumonia, respectivamente. A presença de quadros de diabetes mellitus, demência, doenças cerebrovascular e vascular periférica e doença pulmonar obstrutiva crônica são eventos freqüentemente encontrados nos idosos. Os indivíduos das ILP usualmente apresentam três ou quatro doenças associadas, sendo que o diabetes mellitus está expressivamente presente na proporção de $10 \%$ a 30\%. A incapacidade funcional, com limitações no autocuidado e incontinências fecal e urinária, a desnutrição documentada em 10\% a 50\% dos idosos em ILP, a necessidade de procedimentos invasivos (cateterização urinária, sonda gastroduodenal, traqueostomia), o uso de várias medicações são outras situações comuns relatadas nesses residentes e que aumentam a ocorrência de infecção.

A infecção adquirida na própria instituição é uma das causas mais freqüentes de hospitalização e de mortes nesses pacientes 5 . Um dos motivos é o retardo no diagnóstico e tratamento. A investigação dessa condição nos residentes de ILP é freqüentemente prejudicada pela limitação estrutural e econômica das unidades. Além da dificuldade de realização de exames laboratoriais e radiológicos, as manifestações clínicas do processo infeccioso são comumente atípicas nessa faixa etária. A febre ausente ou baixa é exemplo clássico dessa atipia no paciente idoso.

No presente estudo, a taxa de infecção foi de 63,6\% e a densidade foi de 3,4 infecções por 1.000 pacientes-dia. Outros estudos documentam uma taxa de infecção de $75 \%$ nos indivíduos em ILP com pelo um episódio de processo infeccioso no período de um ano ${ }^{10}$, enquanto a densidade desta condição foi de 4,1 , com variação de 0,8 a 9,5, segundo o nível de dependência da população assistida". Dados mostram que existem semelhanças entre as densidades de incidência de infecção obtidas entre as ILP, mesmo que guardem diferenças socioeconômicas e estruturais. A prevalência das topografias varia conforme as características da população estudada, a metodologia de investigação empregada e o uso de exames subsidiários para a confirmação diagnóstica'2. O presente estudo mostrou que a infecção respiratória foi a mais prevalente (50\%), seguida pela do trato urinário (32\%) e pele e partes moles (11\%). Dentre as infecções mais comuns entre os residentes de ILP são descritas: trato urinário, respiratória, pele e partes moles e gastrintestinais ${ }^{13,14}$

Nosso estudo também identificou que a densidade de infecção por 1.000 pacientes-dia foi de um para trato urinário, I,6 para trato respiratório e 0,4 para pele e partes moles. Estudo de Stevenson ${ }^{15}$ demonstrou que, em 1.000 pacientes-dia de ILP americana, a densidade de infecção de trato urinário foi de |,5।, respiratório I,I5 e gastrintestinal de 0,86. Nossas taxas de infecção em pacientes-dia foram maiores na topografia respiratória e menor na urinária, provavelmente em razão das características clínicas dos pacientes e às condições diagnósticas empregadas nas instituições envolvidas nos estudos.

Estudo prospectivo relatou a taxa de letalidade das infecções de 6,3\% em todos os episódios de infecção ${ }^{16}$. Obtivemos taxa de letalidade de 6,9\%, maior que o relatado na literatura ${ }^{16}$, provavelmente pelas características da população estudada, e maior incidência da infecção respiratória, a qual apresenta a maior taxa de letalidade ${ }^{14}$.

Agentes etiológicos foram isolados em 30\% das infecções, sendo os prevalentes Escherichia coli, Staphylococcus coagulase negativa e Pseudomonas aeruginosa. Os patógenos descritos na infecção respiratória são Streptococcus pneumoniae e Haemophilus influenza ${ }^{4}$. Em nosso estudo, não foi realizado diagnóstico nessa topografia por condições técnicas. $\mathrm{Na}$ infecção do trato urinário, obtivemos etiologia em $47 \%$ dos episódios, sendo os mais freqüentes Escherichia coli e Staphylococcus coagulase negativa, enquanto são descritos como os mais freqüentes Enterococcus faecalis e Escherichia coll ${ }^{4}$. Na infecção de pele e partes moles, obtivemos agentes etiológicos em $44 \%$, sendo os mais freqüentes Staphylococcus coagulase negativa e Pseudomonas aeruginosa, enquanto a literatura relata a ocorrência de Herpes-Zoster e escabiose $^{4}$, as quais não foram observadas em nosso estudo.

\section{ConClusão}

A incidência das infecções observadas nos indivíduos institucionalizados no Asilo Padre Euclides de Botucatu, bem como sua distribuição topográfica, apresentou-se com índices semelhantes ao relatado na literatura, porém com taxa de letalidade maior. A ocorrência desse evento é uma condição clínica importante para essa população, sendo causa freqüente de descompensação e de hospitalização.

Conflito de interesse: não há.

\section{SUMMARY}

\section{INFECTION IN ELDERLY INTERNED IN LONG-TERM CARE FACILITIES}

OBJECTIVE. To evaluate the occurrence of infection in elderly interned in long-term care facilities (LTCF) during 13 consecutive months.

METHODS. Fifty five elderly were evaluated according to infection and, reporting was standardized.

RESULTS. The median age was $75(66-81)$ years. The data set consisted of 20,896 patients-day (PD) of care during the period, with a total of 76 infections for a pooled mean rate of infections 3.20 per 1,000 PD of care. Patient infection rate was 63.6\%. Prevalent topographies of infection were respiratory (50\%), urinary tract (32\%), skin and soft tissue (12\%) and gastroenteritis (7\%), with an infection rate for specific categories/1000 PD of 1.60, 1.01, 0.38, 0.21 , respectively. Mortality infection rate was $5 \%$. Microbiological agents were isolated in 30.5\% of the infection episodes. Escherichia coli and Staphylococcus aureus were prevalent.

CONCLUSION. Infection presented an elevated incidence in the elderly interned, but no unusual problem with infections was disclosed. [Rev Assoc Med Bras 2007; 53(2): 126-9]

KEY WORDS: Elderly. Infection. Epidemiology. Long-term care facilities. 


\section{REFERÊNCIAS}

I. Organización Mundial de la Salud. Grupo Científico sobre la epidemiología del envejecimiento. Ginebra: OMS; 1984. [Serie de informes técnicos, 706].

2. Kemper $P$, Murtauger DM. Lifetime use of nursing home care. N Eng Med. |991;324:595-600

3. Born T. Cuidado com idoso em instituição. In: Papaleo Netto M. Gerontologia: a velhice e o envelhecimento em visão globalizada. São Paulo: Editora Atheneu; 1996. p.403-14.

4. Richards C. Infections in residents of long-term care facilities: an agenda for research. Report of an expert panel. J Am Geriatr Soc. 2002:50:570-6

5. Nicolle LE, Strausbaugh LJ, Garibaldi LJ. Infections and antibiotic resistance in nursing homes. Clin Microb Rev. 1996;9:1-17.

6. Richards CL. Preventing antimicrobial-resistant bacterial infection among older adults in long-term care facilities. J Am Med Dir Assoc. 2005;6: I 445 !.

7. Irvine PW, Van Buren N, Crossley K. Causes of hospitalization of nursing home residents: the role of infection. J Am Geriatr Soc. 1984;32:103-7.

8. Bentley DW, Bradley S, High K, Schoenbaum S, Taler G, Yoshikawa TT. Practice guideline for evaluation of fever and infection in Long-Term Care Facilities. Clin Infect Dis. 2000;31:640-53.

9. Smith PW, Rusnak PG. Infection prevention and control in the longterm-care facility. Infect Control Hosp Epidemiol. 1997; 18:831-49.
10. Yoshikawa TT, Norman DC. Approach to fever and infection in the nursing home. J Am Geriatr Soc. 1996; 44:74-82.

II. Nicolle LE, Garibaldi RA. Infection control in long-term-care facilities. Infect Control Hosp Epidemiol. 1995; 16:348-53.

12. Nicolle LE. Infection control in long-term care facilities. Clin Infect Dis. 2000;31:752-6.

13. Murder RR. Pneumonia in residents of long-term care facility. Am J Med. 1998; 105: 319-30.

14. Furman DV, Rayner AV, Tobin EP. Pneumonia in older residents of long-term care facilities. Am Fam Physician 2004;70:1495-500.

15. Stevenson KB. Regional data set of infection rates for long-term care facilities: description of a valuable benchmarking tool. Am J Infect Control. 1999;27: 20-6.

16. Nicolle LE, McIntyre M, Zacharias H, MacDonell J. Twelve month surveillance of infections in institutionalized elderly men. J Am Geriatr Soc. 1984;32:513-9.

Artigo recebido: 18/04/06

Aceito para publicação: 29// I/06 